\title{
The Operation of Junction Transistors at High Currents and in Saturation*
}

\author{
C.A. Mead
}

Abstract - The lumped model characterization for junction transistors has been extended to current ranges where the transistor operation may no longer be considered linear. A number of effects are considered which contribute to the nonlinear behavior. In all cases it is shown that the self-bias cutoff effect plays an important part in the deterioration of performance at high currents.

In alloy transistors, both normally and inversely biased, an exact solution for the form of the current gain as a function of collector current is given for the case where the injected density is small compared with the equilibrium base majority carrier density. This solution is shown to also be applicable to normally biased diffused base units. A decrease in current gain is predicted due to the self-bias effect. The sources of lateral base current are bulk recombination and injection into the emitter, the relative amount being immaterial since both are proportional to the injected density. In the small area alloy devices, it is seen that the injected density becomes large compared with the base majority carrier density before the transistor performance is appreciably degraded. In such cases, the lateral base current is seen to normally be dominated by non-unity emitter efficiency.

In all cases, surface recombination is assumed to be the dominant source of total base current, and the first order correction to the linear theory is shown to exhibit a $1 /\left(1+K i_{c}\right)$ dependence. At higher currents, all cases follow the observed $1 / i_{c}$ proportionality.

The affect of nonlinear phenomena on saturation voltage is considered in some detail. The saturation voltage is shown to increase with drive current, again emphasizing the importance of avoiding large overdrive currents. The use of alloy transistors as low-level signal switches is discussed, indicating the necessity for high values of $\beta$ and low values of $K$.

\section{Introduction}

The analysis of junction transistor switching performance has required the development of characterization techniques considerably more advanced than those used for small signal analysis. Perhaps the most satisfactory technique which has emerged is that of the linear lumped model [1, 2].

It has been pointed out that the lumped model approach provides a deep understanding of the physical principles underlying transistor operation. One is naturally led to ask if such an approach would also provide a similar understanding of the nonlinear behavior of junction transistors which predominates as current densities are pushed higher and higher. In the sections which follow, the nonlinear theory will be investigated in some detail, and modifications of the linear theory which are necessary under a number of

${ }^{*}$ Re-typeset from original material by Donna Fox, December 2017. Originally published in Solid-State Electronics, Pergamon Press, 1960. Vol. 1, No. 3, pp. 211-224. 
conditions encountered in practice will be established. Throughout the discussion, a p-n-p transistor is assumed in the interest of clarity and simplicity of notation.

The lumped model for a p-n-p alloy transistor with very high conductivity collector and emitter regions is shown in Fig. 1.

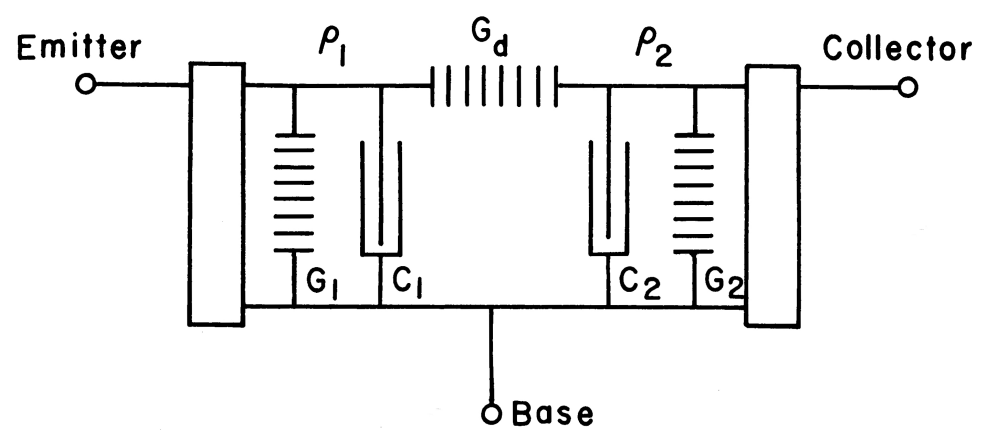

Figure 1: Complete two-section lumped model of junction transistor with high conductivity collector and emitter regions.

The symbols have the same significance as passive electrical circuit elements, except that they relate the excess minority carrier density $\rho$ rather than voltage to the current. In this model $\rho_{1}$ represents the excess density near the emitter, given by

$$
\rho_{1}=P_{n}\left(e^{q v_{e b} / k T}-1\right) .
$$

where $v_{e b}$ is the emitter-base junction voltage; $C_{1}$ and $G_{1}$ represent storage and recombination near the emitter, $G_{d}$ represents diffusion from emitter to collector, $\rho_{2}$ is the excess density near the collector, given by

$$
\rho_{2}=P_{n}\left(e^{q v_{c b} / k T}-1\right)
$$

where $v_{c b}$ is the collector-base junction voltage; $C_{2}$ and $G_{2}$ represent storage and recombination near the collector. Both voltages are taken positive when the junction is forward-biased. A very detailed discussion of the linear lumped model is given in [2].

\subsection{Summary of Nonlinear Effects}

Let us now investigate in general the effects which would be expected to contribute to the nonlinear behavior of a junction transistor. Although a complete list would necessarily be of much greater length than utility, the following effects are of some importance in present devices, and merit careful consideration.

\subsubsection{Depletion layer recombination}

At low injection levels in silicon devices, junction operation is dominated by carrier recombination within the depletion layer. In germanium devices and in silicon devices operating at higher levels, this effect is negligible [3, 8, 9]. Since we are specifically interested in the high-level case, we shall assume the effects of depletion layer recombination are small. However, some discrepancies will be found to exist between the high-level theoretical expressions and data taken at relatively low level, which may be explained in terms of this omission, but which are not important for the purpose of this discussion. The effect of such recombination on the lumped model is to introduce conductances in parallel with $G_{1}$ and $G_{2}$ which decrease rapidly as $\rho_{1}$ and $\rho_{2}$ become appreciable. 


\subsubsection{Drift-enhanced diffusion flow}

When minority carriers are injected into a region of semiconductor, the condition of near-charge neutrality requires an equal number of additional majority carriers. A gradient in excess minority carrier density therefore implies a gradient in majority carrier density. If the majority carrier current is constrained by means of junctions, the diffusion current tendency implied by this density gradient must be canceled by an equal and opposite drift current tendency; hence, an electric field is developed. As long as the injected density is small compared to the equilibrium majority density, the drift component of minority carrier resulting from this electric field is small compared to the diffusion component. However, at high injection levels, the drift component becomes appreciable, resulting in an effective diffusion constant larger than at low densities. At very high levels, the effective diffusion constant approaches twice its low-level value [4]. As we will later separate the high- and low-level cases, it will not be necessary to consider the transition in detail. However, since the low-level limiting form of the solution is of interest, it must be borne in mind that the high-level value of $G_{d}$ will be twice the low-level value due to this effect.

\subsubsection{Recombination rate}

For most purposes, the rate of recombination of minority carriers may be considered proportional to the product of majority and minority carrier densities. However, in semiconductor material as commonly used, recombination occurs principally at traps or recombination centers, and the recombination statistics are altered [8, 9, 17]. This effect is chiefly responsible for the rapid decrease in current gain at low current, but may be neglected at higher current levels.

\subsubsection{Conductivity modulation}

The conductivity of a semiconductor is usually taken as proportional to the equilibrium density of majority carriers which, under normal conditions, is equal to the density of donor or acceptor atoms. However, at high levels the density of majority carriers is increased as the density of minority carriers becomes appreciable. Hence, the number of carriers available for current flow is increased and the conductivity of the material is increased. Ohmic resistance effects (for example, the base resistance and series collector resistance of a transistor) are altered to the extent that these effects arise in the active region of the transistor. The conductivity $\sigma$ of the material becomes

$$
\sigma=\sigma_{o}\left[1+(1+b) \frac{\rho}{n_{n}}\right],
$$

where $\sigma_{o}$ is the low-level conductivity, and $b$ is the ratio of minority to majority carrier mobilities [12].

\subsubsection{Junction injection efficiencies}

When a p-n junction is forward biased, in addition to the holes injected from the p-region into the n-region, electrons are injected from the n-region into the p-region. The ratio of the injected densities in the two regions is inversely proportional to the ratio of the majority carrier densities. If the equilibrium majority density of one region is much greater than that of the other, the injected density will become comparable to the majority density at much lower levels in the low conductivity region. Hence the ratio of injected densities will vary with the injection level. This effect becomes significant, for example, at a forward-biased junction of a transistor. 
Although the contribution to the total base current in a junction transistor due to non-unity injection efficiency is normally small compared with that due to surface recombination, the lateral base current within the active area of the device is often principally due to injection effects and hence of great importance in connection with the self-bias cutoff effect.

As previously noted, in many transistor junctions the conductivity of the transistor base region is relatively low compared with that of the other region in question. In this case, the current density $J$ injected from the base region into the other region may be shown to be related to the excess density $\rho$ in the base region as follows [3, 21].

$$
J \sim \rho\left(1+\frac{\rho}{n_{n}}\right)=P_{n}\left(e^{q v / k T}-1\right)
$$

where

$P_{n}$ is the equilibrium minority carrier density in the base,

$k$ is the Boltzmann's constant,

$T$ is the absolute temperature,

$v$ is the voltage across the junction, being taken as positive when the junction is forward biased.

\subsubsection{Density crowding or self-bias effect}

Because of volume recombination and non-unity junction efficiencies, majority carrier current is caused to flow laterally in the base region. This current must flow through the sheet resistance of the base region and hence causes a lateral potential gradient in the base region. This gradient is in such a direction as to decrease the junction forward bias in the center of the base region. Hence, at high levels the injected carriers are essentially confined to the outer edge of the base region [5, 14, 18]. The major consequence of this effect is a reduction in the effective area of the active region of the transistor. In many transistors, the high-level behavior is dominated by this effect. In this paper, we will consider the results of density crowding in considerable detail.

\subsubsection{Thermal effects}

When a junction transistor is operated at high current densities, heat is evolved at junctions where the applied reverse bias is appreciable and also in the ohmic resistances associated with the various regions. Changes in temperature of the device caused by heat generated in this manner cause changes in the electrical properties of the unit which are often very difficult to distinguish from the high-level phenomena previously discussed. Junctions in a transistor or diode are often assigned a certain thermal time constant and thermal resistance to the mounting base of the device, and it is thus implied that the junction itself possesses a certain thermal capacitance, which may be fairly large. However, the flow of heat in a solid is basically a diffusion process, and it should be recognized that the lumped approximation implied by the thermal time constant and thermal resistance is valid only under conditions where the thermal gradients are not too severe. Under conditions of very high power input for very short periods, such as during pulse measurements, the lumped approximation would predict a very small change in temperature. In reality, since the power is dissipated only within the junction and hence is localized to a very small volume, the actual local temperature may rise to a very high value. Such a temperature increase causes violent changes in the equilibrium minority carrier density as well as less drastic changes in lifetime, mobility, and surface recombination properties. Although we shall not treat these effects in detail, we must always be aware that experimental results may be colored by their presence. 


\subsubsection{Hole-electron scattering}

As the injected carrier density reaches very high levels, a decrease in the mobility of minority carriers is to be expected due to hole-electron scattering. This effect is treated in considerable detail in a paper by Fletcher [16], where the mobility of holes and electrons is plotted as a function of minority carrier excess density. Although these results have not been explicitly incorporated into the present work, it should be noted that since the lumped model approach has been used throughout, the determination of injected densities is a necessary step in the solution of any high-level problem. Like Fletcher [5] and others [14, 18], we have assumed the mobility to be constant. However, a good estimate of the effects of hole-electron scattering may be obtained using the computed values of injected density in connection with Fletcher's data.

\subsubsection{Outline of analysis}

In order to gain a basic understanding of transistor high-current operation, we have briefly considered a number of the more important effects which contribute to its nonlinear behavior. We shall now restrict our attention to a number of specific cases of practical interest.

Of the bewildering array of transistors now available, we shall consider only the alloy transistor, although many of the results are applicable to other types as well. Due to the self-bias effect, we expect nonlinear performance even at injection levels low compared with the majority carrier density. For many alloy units, much of the operation is performed under very high-level conditions. The corresponding analysis must include the effects of decreased injection efficiency and base conductivity modulation, which considerably modify the form of the self-bias effect, and are treated in some detail.

Finally, operation in the saturation region is considered, where the injection efficiency of both emitter and collector is seen to be important. The electric field across the base region due to majority carrier density gradient may also make important contributions to the saturation voltage at high levels.

\subsection{Nonlinear Operation; Normal Bias}

The basic equations describing the operation of a junction transistor under conditions where carrier density crowding must be taken into account will now be derived. The analysis is presented for a normallyconnected transistor, but is equally valid for the inverse connection. A typical transistor geometry is shown in Fig. 2. The structure is assumed two-dimensional, i.e., independent of the coordinate normal to the page. Thus, the analysis will be accurate for stripe and ring configurations and interleaved finger power transistors. It will also be approximately correct for dot configurations at high levels where the current is

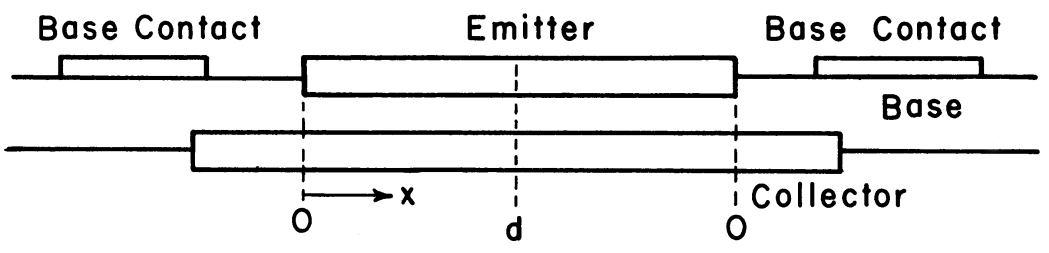

Figure 2: Structure of alloy transistor.

confined principally to the edge of the emitter. Initially, we shall solve for the distribution of minority carrier density in the base region caused by the self-bias effect as a function of the distance from the edge 
of the emitter $x$. This information will allow us to calculate the base and collector current, and hence the behavior of current transfer ratio as a function of current level. Another way of expressing the solution is in terms of the variation of the lumped model parameters as a function of the excess density.

\subsubsection{Low-level approximation}

As noted before, if $\rho \ll n_{n}$, both the electron current injected into the emitter and the bulk recombination may be considered proportional to the excess density. Hence, the lateral ( $x$-directed) base current flowing outward from the interior base region may be written as

$$
i(x)=A \int_{d}^{x} \rho\left(x^{\prime}\right) d x^{\prime}
$$

where $A$ is the sum of two terms, the first due to volume recombination and the second due to non-unity emitter efficiency.

The lateral base current flowing through the sheet resistance of the base creates a lateral voltage drop within the base region. This self-bias voltage drop is in such a direction as to decrease the forward-bias voltage applied to interior portions of the emitter-base diode [5]. The voltage $v(x)$ between the base at the outer edge of the emitter $(x=0)$ and at some interior point $x$ may be written as

$$
-v(x)=R \int_{o}^{x} i\left(x^{\prime}\right) d x^{\prime} .
$$

(Note that $A$ and $R$ may be considered constant only when $\rho \ll n_{n}$; conductivity modulation of the base region and the decrease in emitter efficiency create important variations which will be treated later.)

The effect of the self-bias voltage $v(x)$ is to decrease the excess hole density injected into the base region from its value at $x=0$. As we have restricted our attention to the case $P_{n} \ll \rho \ll n_{n}$, we may write the injected density in terms of the self-bias voltage $v(x)$ from Eq. 4 .

$$
\rho(x)=\rho(0) e^{-q v(x) / k T} .
$$

Both the mobility and diffusion constant of minority carriers have been assumed independent of excess density, as noted earlier. Hence, each volume element may be considered as a separate transistor, and the total diffusion current through each element is proportional to the density gradient, or in the special case where the collector is reversed-biased, to the emitter excess density at the element in question. Integrating the contributions to the diffusion current over all elements, the total collector diffusion current per unit length of the structure $i_{c}$ may be written

$$
i_{c}=F \int_{o}^{d} \rho\left(x^{\prime}\right) d x^{\prime},
$$

where $F$ is the carrier diffusion constant divided by the base width.

The formulation of the basic equations necessary for determining the transistor high-level operating characteristics is now complete. These equations with appropriate modifications will also be used for cases treated in later sections. 
It may be verified by direct substitution that the solution of this set of simultaneous, nonlinear, integral Eqs. 5, 6, and 7 is 14

$$
\begin{aligned}
i(x) & =B \tan \frac{(d-x)}{s}, \\
\rho(x) & =\rho(d) \sec ^{2} \frac{(d-x)}{s}
\end{aligned}
$$

where

$$
\begin{aligned}
B^{2} & =\frac{2 k T \rho(d) A}{q R}, \\
s^{2} & =\frac{2 k T}{\rho(d) A R q} .
\end{aligned}
$$

Since the lateral base current evaluated at the edge of the emitter $i(0)$ is proportional to the area under the density curve, and since the total collector diffusion current $\left(i_{c}\right)$ is also proportional to the area under the density curve, the ratio of the two is a constant,

$$
i_{c}=\frac{F}{A} i(0)
$$

Thus, one might expect the forward current gain $\beta$ of the transistor to be constant. However, it should be recalled that the effects of bulk recombination and emitter efficiency on $\beta$ are normally not appreciable compared with the effect of surface recombination [21]. Even an approximate analysis of the surface recombination current is extremely involved and becomes so entangled with the exact geometry of the specific transistor as to be useless in general. However, it may be shown that for a wide variety of practical cases, the surface recombination may be considered nearly proportional to the excess density at the edge of the emitter [11]. This amounts to assuming $G_{1}$ independent of $\rho$. Hence, if for the purpose of determining the total base current we neglect bulk recombination and the effects of non-unity emitter efficiency, $i_{b}$ is proportional to $\rho_{1}$. We will now investigate the dependence of $G_{d}$ on the injection level. From Eq. 9 we may write

$$
\rho(d)=\rho_{1}-\frac{3}{2} \frac{K}{G_{d 0}} i_{c}^{2}
$$

where

$$
K=\frac{q R A d}{3 k T F}
$$

Let us now investigate the special limiting case where $i_{c}^{2} K$ is very small compared with $\rho(d) G_{d 0}$, and the excess density is uniform across the base region. Under these conditions, $K_{2} d$ is small and we may approximate the tangent by its argument

$$
i_{c} \approx \frac{F}{A} \frac{B}{s} d=F d \rho(d) \approx F d \rho(d) .
$$

Hence, the value of $G_{d}$ at low levels $\left(G_{d 0}\right)$ is seen to be

$$
G_{d 0}=F d
$$

Returning to the more general case, Eqs. 9, 12, and 13 may be written

$$
i_{c}=\sqrt{\frac{2 \rho_{1} G_{d 0}}{3 K}-i_{c}^{2}} \tan \frac{3 K}{2} \sqrt{\frac{2 \rho_{1} G_{d 0}}{3 K}-i_{c}^{2}} .
$$


In order to generalize the result of this analysis, we will express the quantities of interest in dimensionless normalized form

$$
\begin{aligned}
\rho^{*} & =\frac{3}{2} \rho G_{d 0} K, \\
i_{c}^{*} & =\frac{3}{2} i_{c} K, \\
G_{d}^{*} & =\frac{G_{d}}{G_{d 0}} .
\end{aligned}
$$

Using this notation, Eq. 16 becomes

$$
i_{c}^{*}=\sqrt{\rho_{1}^{*}-i_{c}^{*}} \tan \sqrt{\rho_{1}^{*}-i_{c}^{* 2}} .
$$

The radical may be considered as a parameter by which the value of $\rho_{1}^{*}$ and $i_{c}^{*}$ are connected. It is also a significant measure of the extent to which the carriers are crowded to the edge of the emitter. The normalized values, $\rho^{*}$ and $i_{c}^{*}$, of the density and collector current may now be plotted and are related as shown in Fig. 3 .

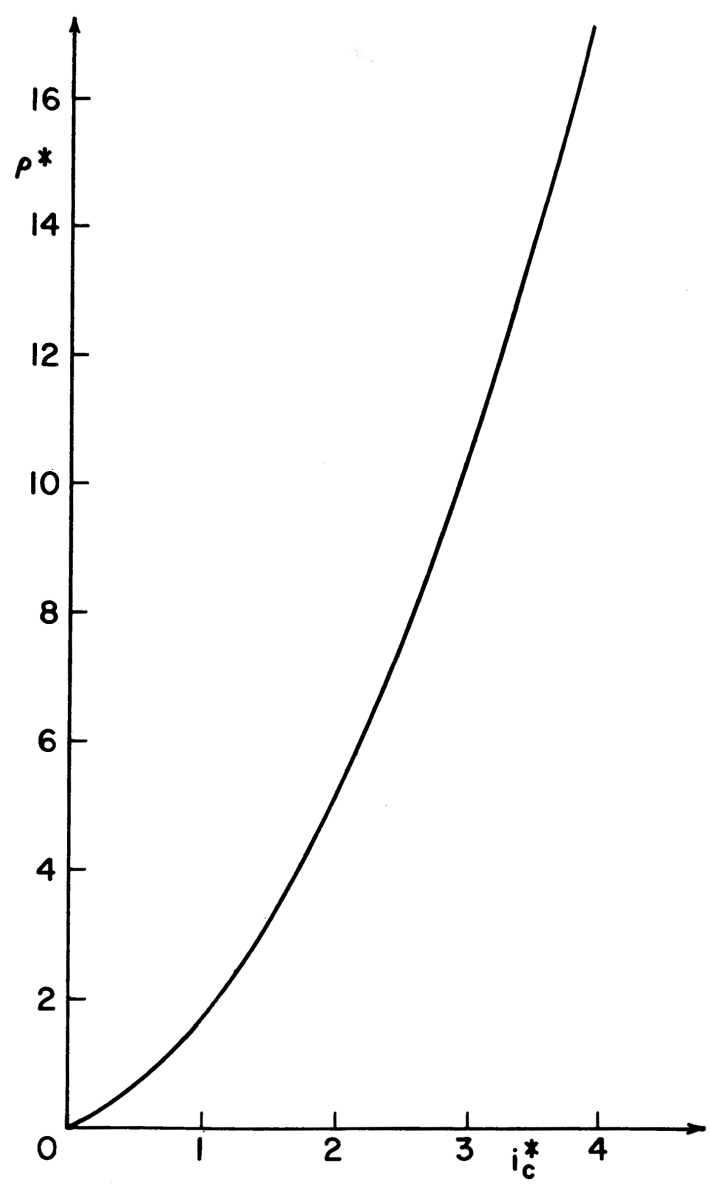

Figure 3: Dependence of collector current upon excess density.

An important implication of these results is the ability to present a unique form for the dependence of $i_{c}$ on $\rho_{1}$ with only one normalizing constant to be determined. As in the linear case, we find the combination $\rho G_{d 0}$ a convenient one with which to deal [2]. 
The relation between $i_{c}^{*}$ and $\rho^{*}$ is seen to be linear at values of $i_{c}^{*}$ much less than unity. At higher levels, $i_{c} *$ increases only as the square root of $\rho^{*}$. The variation of the effective diffusion conductance with excess density and also with collector current is shown in Fig. 4.
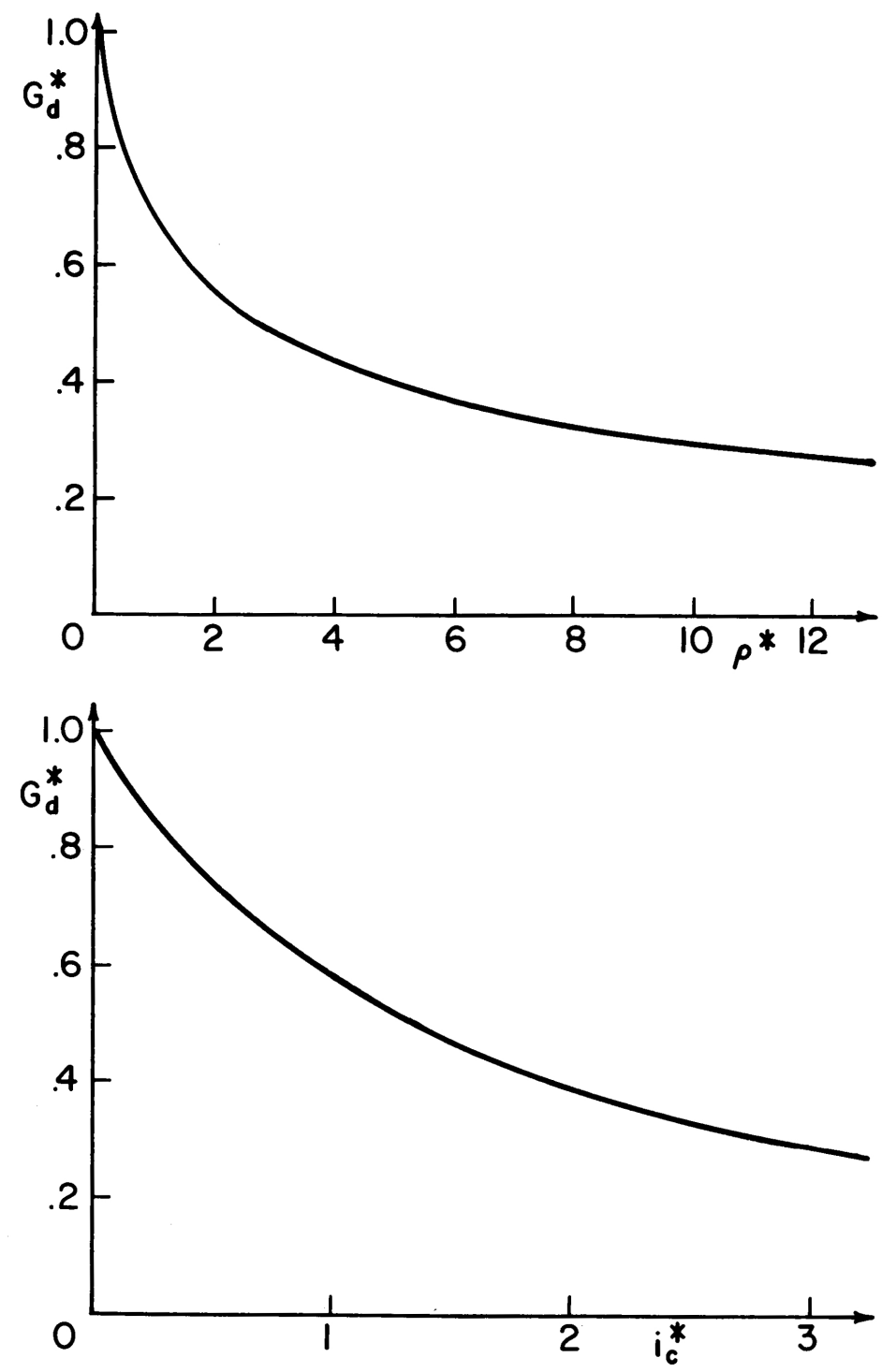

Figure 4: Theoretical variation of effective diffusion conductance.

Again, only the constant $K$ (in addition to the linear lumped model element values) is needed to completely characterize the transistor. The inverse of this constant, given in units of current, is a valuable figure of merit for transistors with which the present approximation is valid. From a circuit standpoint, this value represents the current at which the transistor has begun to fail, and therefore provides a reliable comparison of the current handling capacity of various units.

From a device design point of view, we are interested in the requirements on the physical parameters of a transistor to make the figure of merit as large as possible. Since the figure of merit is inversely proportional to $K$, we should strive for large values of $F$ and small values of $R$, $A$, and $d$.

The decrease in $G_{d}$ at higher levels is due to a decrease in the effective area of the transistor due to the density crowding or self-bias effect. A very large reduction in $G_{d}$ may be observed while the injected 
density is still small compared with the equilibrium majority density, $n_{n}$. In large area power transistors, this condition is very prevalent.

Experimental data taken on an alloy power transistor of essentially two-dimensional construction are shown in Fig. 5, together with the theoretical relation as given in Fig. 3.

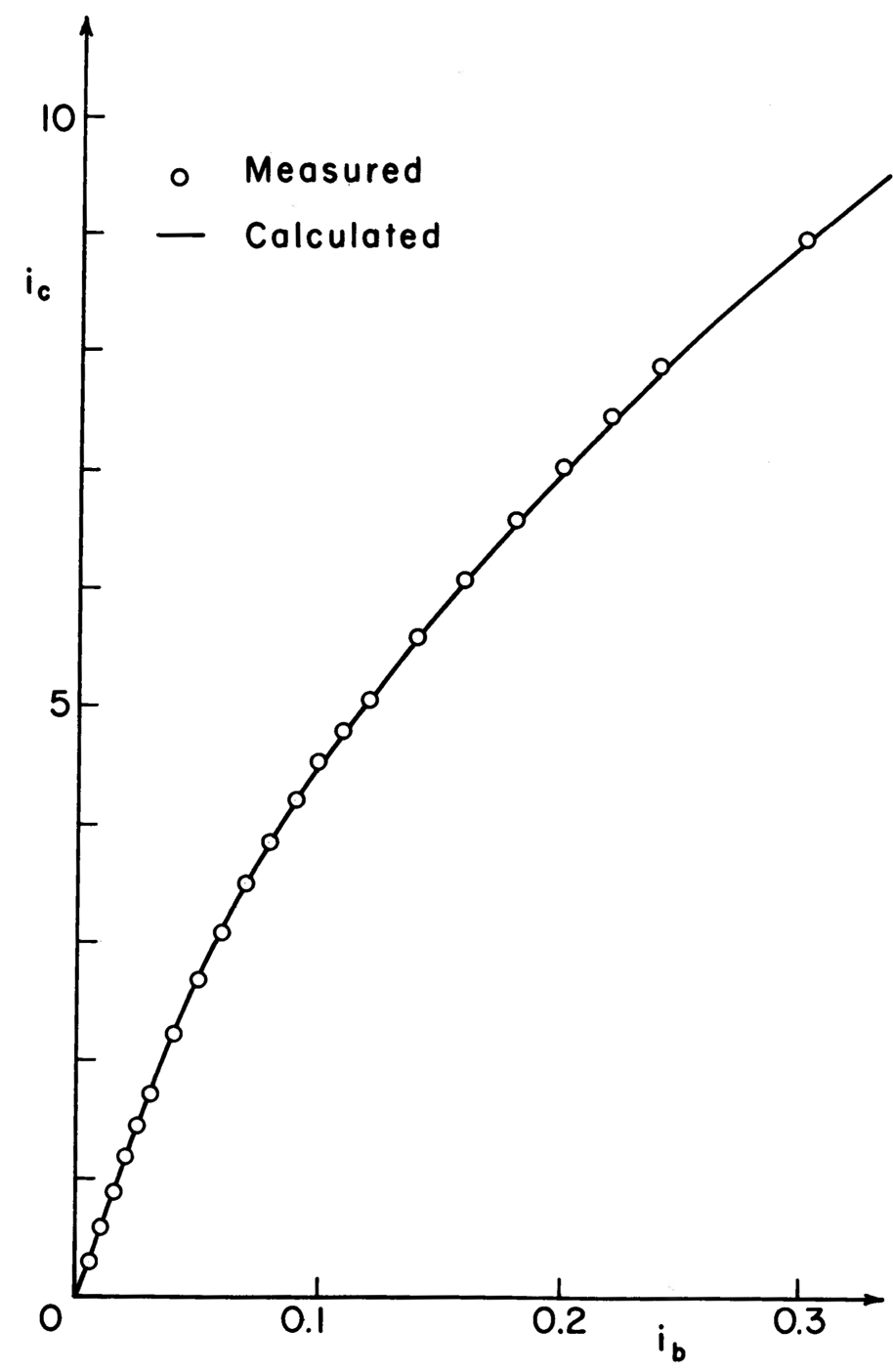

Figure 5: Experimental verification of Fig. 2.

The value of $K$ has been determined to give the best fit. It can be seen that the results agree within experimental error over a very wide current range.

- Near uniform approximation. Closed-form solutions for the relation between $i_{c}$ and $\rho_{1}$ may be obtained for the two limiting cases of very slight and very severe crowding. For the slightly crowded case, the diffusion conductance may be written

$$
G_{d} \approx \frac{G_{d 0}}{1+K \rho_{1} G_{d 0}}
$$

Since the condition for validity of this approximation is that the correction term involving $K$ be small compared to unity, we may obtain an approximate expression for the current gain by substituting

$$
i_{c} \approx \rho_{1} G_{d 0}
$$


in the correction term. Again, the value of $G$ may be assumed constant and the current gain may be written

$$
\beta \approx \frac{\beta_{o}}{1+K i_{c}} \approx \beta_{o}\left(1-K i_{c}\right)
$$

This expression is very significant since it gives the form of the falloff of $\beta$ in the most useful range of operation.

- Severely crowded approximation. Under conditions of severe crowding, the injected density and collector current are related by the following expression

$$
\rho_{1}^{*} \approx i_{c}^{*}+\frac{w^{2}}{4} \quad \rho^{*} \gg 1
$$

In the range where this approximation is valid, the constant term may be neglected without appreciably affecting the accuracy. Hence, the diffusion conductance may be written

$$
G_{d}^{*} \approx \frac{1}{i_{c}^{*}},
$$

or

$$
G_{d} \approx \frac{2 G_{d 0}}{3 K i_{c}}
$$

which implies:

$$
\beta \approx \frac{2 \beta_{o}}{3 K i_{c}}
$$

It is interesting that this expression agrees with the high current limit of Eq. 25 within the factor of $3 / 2$, and the conclusions to be drawn from either expression are the same.

A plot of $\rho^{*}(0)$ as a function of $i_{c}^{*}$ is shown in Fig. 6, together with the asymptotic forms at low and high currents.

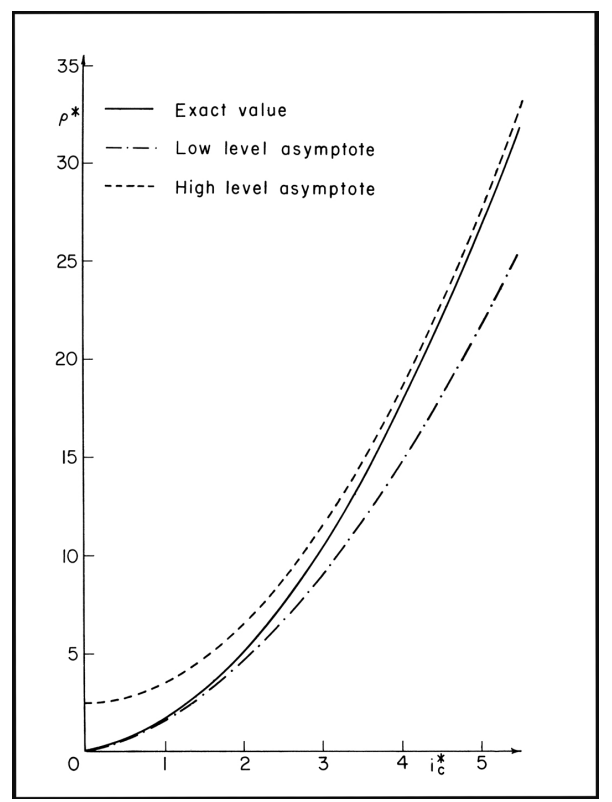

Figure 6: Asymptotes to the relation of Fig. 3 for slight and severe crowding. 
It may be seen that results obtained by means of the asymptote are never in error more than about 10 percent, the worst case occurring when $\rho^{*} \approx 10$ and convergence is quite rapid at lower or higher values.

- High-level approximation. The foregoing expression for the figure of merit indicates that the self-bias cutoff effect or emitter density crowding as discussed would be most noticeable in transistors with large emitter dimensions, namely, power transistors. Indeed, the effect was first treated in detail by Fletcher [5] in connection with power transistors. However, his analysis assumed infinite emitter width and hence did not treat the transition from uniform flow to the crowded condition. It is clear from Eqs. 12, 13, and 25 that for a given emitter area, the current carrying capability varies inversely with the emitter width. Hence, the most efficient design for such units is an interleaved system of narrow base and emitter stripes or rings [5, 12, 13]. As the emitter width is decreased, the self-bias effect becomes less noticeable and current densities are pushed higher and higher. In many transistors now available, the assumption of $\rho \ll n_{n}$ is very poor, in fact the other extreme $\rho \gg n_{n}$ is often valid over the greater part of the range of operation. Thus, we must re-examine the analysis including the effects of large injected density.

At high injection levels, two major changes in the physical operation occur:

- The emitter efficiency decreases as shown in Eq. 4. This decrease may be quite large, and in general at high levels the current injected into the emitter region will be the dominant source of lateral base current inside the area covered by the emitter.

- The resistivity of the base region decreases due to conductivity modulation caused by the injected minority carriers, as shown in Eq. 3. Hence $R$ may not be considered a constant, and the variation with $\rho$ must be shown in the integral.

If we assume that all of the lateral base current is due to the decrease in emitter injection efficiency and neglect bulk recombinations, we may directly apply the expression for current density given in Eq. 4. The expression for lateral base current then becomes

$$
i(x)=A \int_{d}^{x} \rho\left(x^{\prime}\right)\left[1+\frac{\rho\left(x^{\prime}\right)}{n_{n}}\right] d x^{\prime} .
$$

The lateral voltage drop caused by this current is also affected by the conductivity modulation of the base region. Since the effective resistivity of each element of base region is reduced as given by Eq. 3 , we may write

$$
-v(x)=R \int_{o}^{x} \frac{i\left(x^{\prime}\right)}{1+\frac{(1+b) \rho\left(x^{\prime}\right)}{n_{n}}} d x^{\prime} .
$$

The junction injection law is also modified at high levels in accordance with Eq. 4. We may assume as before $\rho \gg P_{n}$

$$
\rho(x)\left[1+\frac{\rho(x)}{n_{n}}\right]=\rho(0)\left[1+\frac{\rho(0)}{n_{n}}\right] e^{-q v(x) / k T} .
$$

Further analysis becomes very difficult, unless another simplification is achieved. Since we are primarily interested in the high current or high injection level limit on the devices, and since $\rho$ becomes equal to $n_{n}$ at quite low current levels in many devices [8, 9], we will make the assumption of high injection level $\left(\rho \gg n_{n}\right)$, realizing that the results will break down at the lower levels, where the previous analysis may be applied. As is often the case with engineering approximations such as these, the regions of mathematical validity for the two approximations are widely separated. However, in practice, the approximations are reasonably good into the intervening range and quite a good representation of the overall performance 
may be made by the use of these relatively simple cases. Eqs. 26, 27, and 28 may be converted to the single differential equation

$$
\frac{d^{2} \rho(x)}{d x^{2}}=\frac{q R A}{(1+b) k T} \rho(x)^{2} .
$$

Two approximate solutions of this equation will be considered.

- Near uniform approximation. The first solution is derived for conditions where the crowding is not severe. In this case, it is shown that the diffusion conductance may be written

$$
G_{d}=\frac{G_{d 0}}{1+K \rho_{1} G_{d 0}}
$$

where in this case the current falloff factor $K$ becomes

$$
K=\frac{q R A d}{3(1+b) k T F},
$$

which is seen to differ from the low-level case of Eq. 19 only by the constant $(1+b)$. The fact that a unique form is available for the two cases is quite significant for the circuit engineer. It permits the nonlinear characterization of the transistor to first order without knowledge of whether the unit is operating at high or low levels, simply by a measurement of the constant $K$.

- Severe crowding approximation. In the case of transistors with wide emitters, severe crowding already exists as $\rho$ becomes comparable with $n_{n}$. If we may assume severe crowding, an exact solution to Eq. 29 is possible.

$$
i_{c}=2 F \sqrt{\frac{6(1+b) k T}{q A R} \rho_{1}}
$$

which indicates a value of $G_{d}$ which is inversely proportional to the collector current. The base current may be written in the form

$$
i_{b}=\rho_{1}\left(G_{s}+\frac{A}{5 n_{n}} \sqrt{\frac{q R A}{6(1+b) k T}} \rho_{1}^{3 / 2}\right),
$$

where $G_{s}$ is the effective surface recombination conductance. As noted before, in most cases the surface recombination term will dominate until very high levels are reached. Under these conditions, $\beta$ is again proportional to $1 / i_{c}$ as in the other cases.

It should be pointed out that in many transistors, notably modern alloy units, where the base resistivity is quite high and the emitter is very heavily doped, the approximations of $p \gg n_{n}$ in the base, and $\eta \ll P_{p}$ in the emitter are valid over a very wide current range, and hence the analysis assumes a greater importance. However, in other cases where the ratio of the conductivities in the two regions is not nearly so large, the operation may vary smoothly from the low-level case to the condition of high injection in both regions.

It seems unnecessary to deal with the correlation between emitter efficiency and current gain falloff as widely treated in the early literature. However, because of the widespread misconception of the principles involved, perhaps a word here is in order. From the results of the foregoing analysis, it is clear that the $1 / i_{c}$ dependence of $\beta$ which was observed is to be expected as a result of the self-bias cutoff effect when the base current is completely dominated by surface recombination. Since the surface recombination is 
known to dominate in a great many transistors, especially earlier units, it is inconceivable that the base current due to decreased emitter efficiency could have been responsible for the rapid falloff in $\beta$, which was observed. The great improvement which was effected by the use of highly doped emitter materials was due to the decrease in lateral base current and hence a decrease in the self-bias cutoff effect, which resulted in a more uniform distribution of carriers across the emitter surface. This improvement resulted in a marked increase in collector current for a given value of injected density. In other words, the observed variation in $\beta$ was primarily the result of a decrease in $G_{d}$ due to emitter crowding, not an increase in $G_{1}$. The necessity for an extremely large value of emitter efficiency is now apparent, since the small internal lateral base current is much more important than previously recognized.

As mentioned before, the results of this section are directly applicable to alloy transistors operating in either the normal or inverse connection; the low-level expressions are also applicable to normally-biased diffused base units. However, it should be noted that many alloy structures utilize a different doping material for the collector junction than for the emitter, and therefore the constant $A$ will not in general be the same for the two connections. Also, the collector is normally considerably larger than the emitter, making the surface recombination conductance $G_{2}$ larger than $G_{1}$.

\subsection{Saturated Conditions; Alloy Transistor}

One of the most outstanding and useful properties of a junction transistor is its unique ability to saturate 1 . Under saturated conditions, the minority carrier density at the collector builds up to the point where the gradient of minority carriers is just sufficient to supply the necessary collector current. As the density curve rises higher, the saturation voltage becomes lower. The limit is reached when the total recombination in the base and injection into the emitter and collector becomes equal to the base-driving current. In the linear theory, the exact source of the base current was not important. However, as high-level conditions are reached, the self-bias effect crowds the carriers toward the outer edge of the collector and hence a large part of the collector junction may be essentially cut off. A rapid increase in base current for a given degree of saturation is caused by the large exposed collector area which has no facing emitter junction, and hence carriers are injected only to be lost in the base region [6].

With the alloying techniques in common use, the base region outside the emitter is considerably wider than inside the active area of the device, and crowding effects may be neglected in this region. Suppose both emitter and collector junctions are forward-biased such that at $x=0$ their respective injected densities are $\rho_{1}$ and $\rho_{2}$. The analysis of this case is much the same as that used in connection with the normally biased transistor. Under the assumption that the value of $A$ is the same at emitter and collector, the results obtained indicate a behavior of $G_{d}$ which is identical with that given in the normal biased case, provided $\rho$ is replaced by $\rho_{1}+\rho_{2}$. As before, two special cases are considered: the near uniform, and severely crowded approximations. In the former case, a closed form solution for the saturation voltage as

\footnotetext{
${ }^{1}$ The saturation voltage of alloy transistors has been analyzed in great mathematical detail by Huang [20. Unfortunately, the two most important features of saturation operation were treated in a self-inconsistent manner. In the determination of the internal lateral base current, the effects of non-unity emitter efficiency were neglected, yet were included later in the calculation of the total base current. Clearly, if this contribution were at all important in the total base current, it would be the dominant source of lateral base current. The second inconsistency occurs in the solution for the radial distribution of carrier density assuming the collector and base radii to be equal (a case which is in general uninteresting). The results obtained under these assumptions are then extended to the case where the radii are not equal. For any case where the crowding is at all severe, this extension is grossly in error. In light of these errors, the value of Huang's analysis is seriously questionable.
} 
a function of $i_{c}$ and $i_{b}$ may be obtained.

$$
v_{s a t} \approx \frac{k T}{q} \ln \left[1+\frac{i_{c}\left(\beta+\beta_{i}\right)+\beta i_{b}}{\beta_{i}\left(\beta i_{b}-i_{c}\right)}(1+c)\right],
$$

where the correction term

$$
c=K \frac{i_{c}\left(\beta-\beta_{i}\right)+\beta i_{b}\left(2 \beta_{i}-1\right)}{\beta+\beta_{i}}
$$

has been assumed small, and $\beta$ has been assumed large compared with unity. In this expression, $K$ is the current amplification falloff factor as given by Eqs. 12 and 13 . It should be noted that ohmic resistances of the collector and emitter are usually negligible, and hence the saturation voltage given by Eq. 34 should be quite accurate. However, in calculating the individual junction voltages, the $i_{b} R_{b}$ voltage may be dominant, and should always be taken into account.

- Low-level switching operation. One of the most important uses of transistors under the conditions of the present approximation is that of a low-level signal switch. For this purpose, a transistor is universally used in the inverted connection because of the lower saturation voltage obtainable. In this application, the emitter current is very small and the transistor is used merely to transfer voltage information from collector to emitter. If the transistor is to operate with any reasonable success in such a connection, $\rho_{1}$ and $\rho_{2}$ must be very nearly equal, and we may greatly simplify the analysis. From the linear lumped model, the saturation voltage under these conditions is predicted to be

$$
v_{s a t} \approx \frac{k T}{q \beta}
$$

However, such operation is quite sensitive to the crowding or self-bias cutoff effect. If we assume $\rho_{1} \approx \rho_{2} \approx \rho$ and $i_{e} \ll i_{b}$, the base current may be written

$$
i_{b} \approx \rho G_{d 0}\left(\frac{1}{\beta}+\frac{1}{\beta_{i}}\right) .
$$

The saturation voltage may be evaluated from Eq. 34, interchanging the inverse and normal parameters, neglecting the $i_{e}$ terms and assuming $\beta \gg 1$.

$$
v_{\text {sat }} \approx \frac{k T}{q} \ln \left(1+\frac{1+c}{\beta}\right),
$$

where

$$
c \approx \frac{2 K \beta \beta_{i} i_{b}}{\beta+\beta_{i}}
$$

Since it is desired to obtain the lowest possible saturation voltage, operation at low base currents is seen to be desirable. However, a lower limit is imposed by the junction depletion layer recombination which causes $G_{1}$ to increase at low current levels and therefore results in an increased saturation voltage.

Operation in the region where depletion layer recombination is important is not recommended for another very important reason. The effective $G_{1}$ due to this effect and hence the effective $\beta$ is very dependent upon temperature. Hence, the saturation voltage stability with temperature at small drive currents may be much worse than at higher drive currents. Transistors made of material free from dislocations and extraneous impurities are much superior in this respect. 
In any transistor which is at all suitable for this type of operation and which is driven with reasonably small base current, the second term in Eq. 38 will be small compared to unity. Thus, the logarithm may be expanded in terms of its argument, and Eq. 38 becomes

$$
v_{s a t} \approx \frac{k T}{q \beta}\left(1+\frac{2 K \beta \beta_{i} i_{b}}{\beta+\beta_{i}}\right) .
$$

where again the correction term has been assumed small compared with unity.

Experimental values of $v_{\text {sat }}$ for an alloy transistor operating at low level are shown in Fig. 7. The

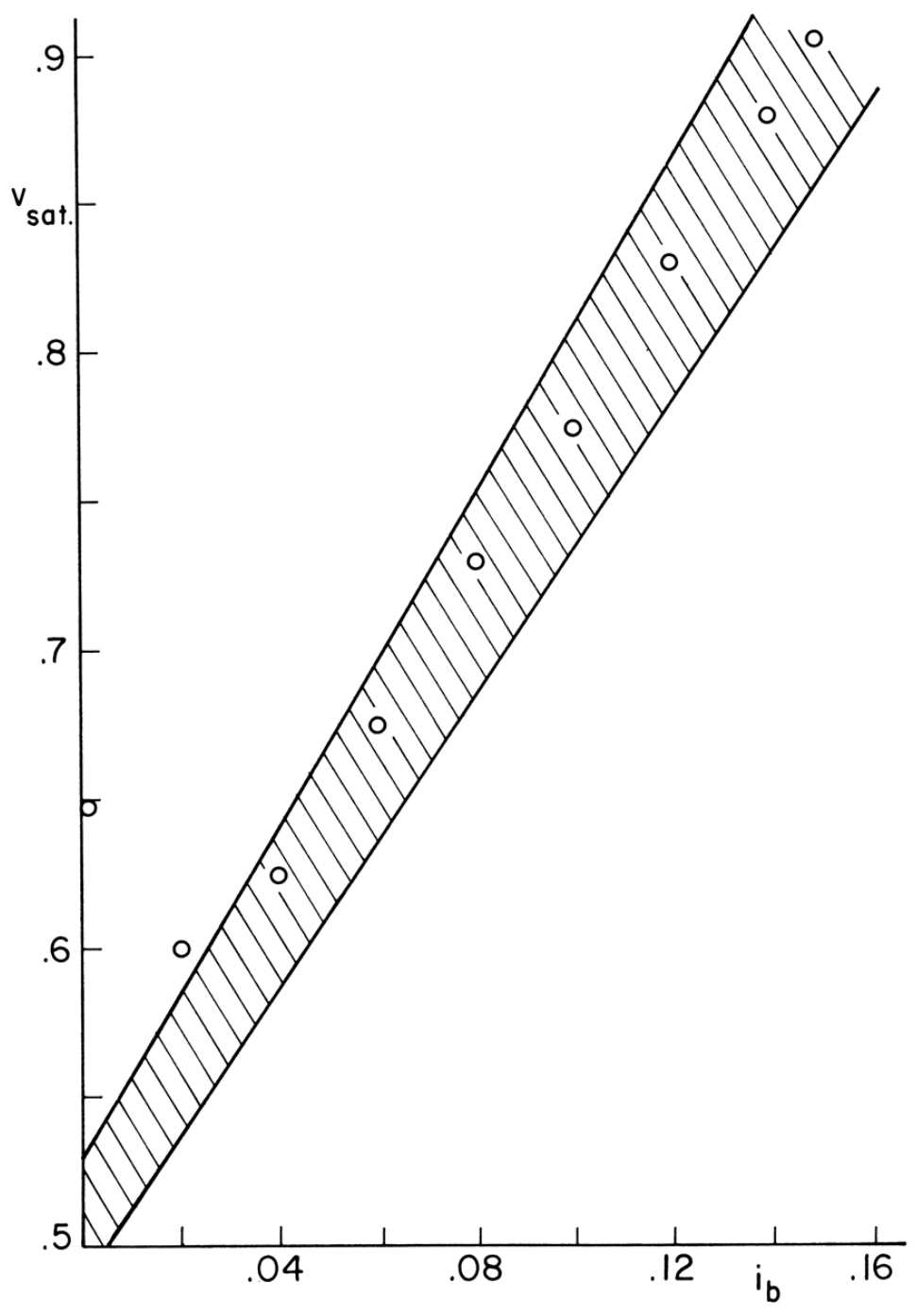

Figure 7: Experimental verification of Eq. 40.

theoretical values fall somewhere within the limits shown depending upon the exact values of $\beta$ and $\beta_{i}$ used in the calculation. The analysis is especially critical to the precise value of $\beta_{i}$ used. Perhaps the most satisfactory method of obtaining $\rho_{1}$ and $K$ is to fit the $i_{e}$ versus $i_{b}$ data to Fig. 3, and use the normalization constants to determine these parameters. The true low-level value of $\beta_{i}$ is often masked by depletion layer recombination at low operating currents. This effect is quite noticeable in the experimental data of Fig. 7, where the saturation voltage increases slightly at low base currents.

The general requirements on a transistor to be used in low-level switching service are that the saturation voltage should be as independent of time, temperature, and variations in drive current as 
possible. Thus, for efficient operation in this type of application, the transistor should have a very high $\beta$ and low value of $K$. Also, the depletion layer recombination should not become important until very low levels are reached.

An obvious extension of the transistor signal switch is to operate two such switches back-to-back so that their saturation voltages tend to cancel. This procedure has often been used with some success. The problems involved are primarily those of matching $\beta$ between units, although the variation of $v_{\text {sat }}$ with drive current is often objectionable and a matching of the drive correction factor may also be attempted, with the obvious increase in labor involved. Again, it should be noted that the depletion layer recombination rate is extremely variable between units, and hence operation in the region where this condition is important is even more to be avoided.

The potential applications of transistor low-level signal switches are very numerous and exciting. In nearly all such applications, the requirements are sufficiently critical that matched back-to-back pairs are desirable. It would seem highly desirable that optimally designed matched units of the same package, mounted on the same mounting base, should be made available to the circuit designer in the same way that avalanche reference elements have been in the past. Perhaps one reason for the overlong delay is the general lack of understanding of the principles underlying the operation of devices used in this type of service. It is hoped that this paper will help to clarify such operation and hasten the day when transistors will replace mechanical elements in many low-level signal switching applications.

- Very high level. If we attempt to solve for the saturated density distribution under very high-level conditions by returning to the initial integral equations, we are faced with an extremely difficult problem. The introduction of the second variable $\rho_{2}$ introduces tremendous complications into the mathematical manipulation and renders a closed-form solution impossible. However, before giving up all hope and resorting to numerical methods, let us consider the real problem with which we are faced. We have derived expressions for the saturation voltage under the assumption that the lumped model elements were linear, and have seen how correction terms were added even at low levels due to the self-bias effect. However, as we have noted, in transistors designed to eliminate the density crowding, (i.e., those with thin emitter stripes) the injected density may well become comparable with $n_{n}$ at current levels where $G_{d}$ has not decreased appreciably below its low-level value. In switching service, such a unit will nearly always be used in the very high-level region, and we are interested in determining the first order correction to the linear theory under these conditions. As an approximation, we will use the variation of $G_{d}$ with $\rho$ as given in Eq. 30 . Since this variation of $G_{d}$ with $\rho$ is of the same form as in the low-level case, we will assume the previous analysis applies and may use the results with the following modifications.

- In units where $\rho$ becomes comparable with $n_{n}$ at reasonably low currents, the value of $\beta$ as measured or given by the manufacturer is normally twice the low-level value due to the drift enhancement of $G_{d}$ mentioned earlier. Since at higher levels this effect is still present, we do not need to add any additional factors to the analysis, but need only be sure that the value of $\beta$ has been measured at a high enough current that the drift enhancement has become effective.

- An additional term must be added to the saturation voltage due to the electric field in the base region caused by the gradient of majority carrier density. Under high-level conditions, the total potential drop across the base region is given by the condition that the drift and diffusion tendencies of majority carrier current are equal.

$$
\mu n E=D \frac{d n}{d y}
$$


This electric field, when integrated across the base region, results in a potential

$$
V=\frac{k T}{q} \ln \frac{\rho_{1}}{\rho_{2}}
$$

Since this voltage is identical to the difference in forward-bias voltages of the junctions and of the same polarity, the effect of high injection level is to double the observed saturation voltage over that given in the low-level case.

- It should again be emphasized that the present analysis has not included the effects of electron-hole scattering. The decrease in $G_{d}$ due to this effect will become appreciable at extremely high levels, and additional saturation voltage is to be expected.

The results of this paper are quite significant to the circuit engineer in that they permit the first order characterization of transistor switches by the use of only one additional parameter, i.e., $K$ the current amplification falloff factor. This modification to the basic theory permits calculations of many switching problems of interest to engineering accuracy. In addition, the author has endeavored to preset a clear physical insight into the fundamental limitations of the device so necessary for complex circuit work. 


\section{References}

[1] Linvill, J.G., "Lumped Models of Transistors and Diodes," Proc. IRE, Vol. 46, (June 1958), p. 1141.

[2] Mead, C.A., "Transistor Switching Analysis," Semiconductor Products, Vol. 3(9), pp. 43-47, 1960 (Part 1); Vol. 3(10), pp. 38-42, 1960 (Part 2); and, Vol. 3(11), pp. 28-32, 1960 (Part 3).

[3] Moll, J.L., "The Evolution of the Theory for the Voltage-Current Characteristic of P-N Junctions," Proc. IRE, Vol. 46, (June 1958), p. 1076.

[4] Webster, W. M., "On the Variation of Junction Transistor Current Amplification Factor with Emitter Current," Proc. IRE, Vol. 42, (June 1954), p. 914.

[5] Fletcher, N.H., "Some Aspects of the Design of Power Transistors," Proc. IRE, Vol. 43, (May 1955), p. 551; Fletcher, N. H., "Self-bias Cutoff Effect in Power Transistors," Proc. IRE, Vol. 43, (November 1955), p. 1669.

[6] Rudenberg, H. G., "On the Effect of Base Resistance and Collector-to-Base Overlap on the Saturation Voltages of Power Transistors," Proc. IRE, Vol. 46 (January 1958), p. 1304.

[7] Rittner, E.S., "Extension of the Theory of the Junction Transistor," Phys. Rev., Vol. 94, (June 1954), p. 1161.

[8] Sah, C.T., R.N. Noyce, and W. Shockley, "Carrier Generation and Recombination in P-N Junctions and P-N Junction Characteristics," Proc. IRE, Vol. 45, (September 1957), p. 1229.

[9] Shockley, W., and W.T. Read, Jr., "Statistics of the Recombinations of Electrons and Holes," Phys. Rev., Vol. 87, (September 1952), p. 835.

[10] Moore, A.R., and J.I. Pankove, "The Effects of Junction Shape and Surface Recombination on Transistor Current Gain. Part I," Proc. IRE, Vol. 42, (June 1954), p. 907.

[11] Stripp, K.F., and A.R. Moore, "The Effects of Junction Shape and Surface Recombination on Transistor Current Gain. Part II," Proc. IRE, (July 1955), p. 856.

[12] Hall, R.N., "Power Rectifiers and Transistors," Proc. IRE, Vol. 40, (November 1952), p. 1512.

[13] Clark, M., "Power Transistors," Proc. IRE, Vol. 46, (June 1958), p. 1185.

[14] Looney, D.H., "Base Resistance Considerations in Transistor Design," Ch. 8, p. 76, Bell Telephone Labs, 14th Interim Technical Report on Transistor Services, (January 1955).

[15] Shockley, W., "The Theory of P-N Junction in Semiconductors and P-N Junction Transistors," Bell System Technical Journal, Vol. 28, (July 1949), p. 435.

[16] Fletcher, N.H., "The High Current Limit for Semiconductor Junction Devices," Proc. IRE, Vol. 45, (June 1957), p. 862.

[17] Hall, R.N., "Electron-Hole Recombination in Germanium," Phys. Rev., Vol. 87, (July 1952), p. 387.

[18] Mortenson, K.E., "High-level Transistor Operation and Transport Capacitance," IRE Trans. on Electron Devices, Vol. ED-6, (April 1959), p. 174.

[19] Griffith, P.G., "Lumped Models of Drift Transistors for Large Signals," Stanford Electronics Laboratories Technical Report No. 1501-1, (April 20, 1959). 
[20] Huang, C., "A Physical Theory of Junction Transistors in the Collector-Voltage-Saturation Region," IRE Trans. on Electron Devices, Vol. ED-6, (April 1959), p. 141.

[21] Middlebrook, R.D., An Introduction to Junction Transistor Theory, John Wiley and Sons, Inc., New York, NY, (1957). 